\title{
Prediction of Skin Lesions in Patients Undergoing Allogeneic Hematopoietic Stem Cell Transplantation Using Generalized Additive Models
}

\author{
Satoko Ueki ${ }^{1}$, Masaaki Tsujitani ${ }^{2}$, Yumiko Teranishi ${ }^{1}$, Junko Miyamoto ${ }^{1}$, Reiko Mori ${ }^{3}$, Katsuji \\ Kaida $^{4}$, Takayuki Inoue ${ }^{4}$, Hiroyasu Ogawa ${ }^{4}$, and Kazuhiro Ikegame ${ }^{4}$ \\ ${ }^{1}$ Central Division of Nursing, Department of Internal Medicine, Hyogo College of Medicine, Hyogo, Japan \\ ${ }^{3}$ Department of Clinical Psychology, Department of Internal Medicine, Hyogo College of Medicine, Hyogo, Japan \\ ${ }^{4}$ Division of Hematology, Department of Internal Medicine, Hyogo College of Medicine, Hyogo, Japan \\ ${ }^{2}$ Graduate School of Engineering, Division of Information and Computer Sciences, Osaka Electro-Communication \\ University
}

\begin{abstract}
In this study, we established a predictive regression model using generalized additive models (GAM) to predict the incidence of skin lesions (SLs) in patients undergoing allogeneic hematopoietic stem cell transplantation (SCT). Among 81 patients who underwent SCT in the SCT unit of the Hyogo College of Medicine between April 2012 and March 2013, 28 developed SL (SL group), and the remaining 53 did not (control group). We defined the following events as states in our multistate model: Diarrhea, need for oxygen supply, hemorrhagic cystitis, skin graft-versus-host disease, encephalitis, and disease relapse. Of these events, diarrhea, need for oxygen supply, and hemorrhagic cystitis occurred more frequently in the SL group than in the control group. A comparison of the function independence measure score revealed more severe muscle weakness in the SL group. A logistic regression analysis using GAMs verified that SL development could be predicted based on serum albumin, blood sugar levels, daily activity scores, and post-transplant day. Of these four predictive covariates, only the post-transplant day exhibited a non-linear curve, with a susceptible peak at approximately 30 days after SCT. GAMs may be a powerful tool for prediction analyses involving time-dependent and non-linear covariates. Patient characteristics may also affect the SL development.
\end{abstract}

Keywords: Skin Lesions, Transplantation, Generalized Additive Models

\section{INTRODUCTION}

Skin lesion (SL) is one of the major issues encountered by patients undergoing allogeneic stem cell transplantation (SCT). This condition not only results in morbidity and mortality but also necessitates considerable health care expenses and nursing manpower. A pressure ulcer is defined as a localized injury to the skin and/or underlying tissue that occurs most often over a bony prominence and is caused by pressure, shear, or friction. In the SCT setting, skin erosions in the perianal or genital area are not necessarily caused by pressure but by fecal incontinence because of gut graft-versus-host disease (GVHD) or other pathogeneses. Because these SLs are difficult to treat after their occurrence, the ability to predict SL development may be beneficial because it facilitates an opportunity to provide appropriate intervention before clinical manifestation. Regarding pressure ulcers, a number of trials on prediction and prevention have been reported in community, 1 nursing home, 2 medical and/or surgical,3 neurological,4 pediatric,5 traumatological,6 and intensive care unit settings,7 whereas SCT unit-based reports are limited. One of the characteristics and difficulties of SCT unit-based studies is the marked dependence of predictive covariates on

${ }^{1}$ Corresponding Author: kame@hyo-med.ac.jp 
the post-transplant day, in addition, the probability of the occurrence of an event such as SL does not always exhibit a linear relationship with the post-transplant day. Although simple linear logistic regression analyses, which are included in generalized linear models (GLMs), have been used in studies in the other abovementioned fields,3,7 these models are unable to account for non-linear covariates; thus, they are inappropriate in an SCT setting.

Generalized additive models (GAMs), or extended GLMs for non-linear curves, were originally applied to address big data-related issues in social sciences. Since then, GAMs have been applied in the biological, 8 environmental, 9 epidemiological,10 and clinical fields.11 We recently extended the theoretical background of GAMs.12,13 In this study, we aimed to use GAMs to establish a model predictive of the occurrence of SLs in patients undergoing allogeneic SCT, and to identify the significant risk factors associated with SL development.

\section{METHODS}

\subsection{Setting}

Hyogo College of Medicine (HCM) is a general hospital in Japan with 963 beds. In our SCT, we perform approximately $80-100$ allogeneic SCTs per year. According to our institutional policy, more than $80 \%$ of patients undergo haploidentical SCT in our unit.

\subsection{Patients And Medical Chart-Based Review}

This retrospective study involved 98 consecutive patients who underwent allogeneic SCT in the SCT unit at HCM between April 2012 and March 2013. Patients who succumbed to early death (within 30 days) after SCT were excluded. If a patient underwent two transplants within the observation period, the first SCT was excluded. The remaining 81 patients (age range: $16-70$ years old, median age $=40$ years) were eligible for this study. Among these patients, 28 patients developed SL (SL group) and 53 did not (Control group). SLs were observed around the gluteal cleft and buttocks, and were confirmed from digital images taken by nurses and recorded in each patient's medical chart. Nurses collected information regarding the patients' variables from medical charts according to the following statistical methods.

\subsection{Daily Activity Score (DAS)}

The DAS was originally established by the Ministry of Health and Welfare in Japan to assess the degree of independence of bedridden and demented elderly individuals, and it has prevailed in Japan as a nursing tool for assessing the degree of patient activity in various clinical fields. The criteria comprise four ranks: Rank J: in which the patient may have some disability, but can perform daily living activities independently, and go out unassisted; rank $\mathrm{A}$, in which the patient can live independently in a house, but cannot go out without assistance; rank B, in which the patient lives in a house and requires some assistance with daily living activities, mainly stays in bed during the daytime, and can maintain a sitting position; rank $\mathrm{C}$, in which the patient is fully bedridden, and requires assistance with excretion, eating, and changing clothes. In this study, DAS was treated as ordinal covariates, and the ranks were ordered as follows: $\mathrm{J}>\mathrm{A}>\mathrm{B}>\mathrm{C}$.

\subsection{Functional Independence Measure (FIM) Score}

The FIM assesses cognitive function, the ability to communicate, mobility, and self-care skills. Eighteen scales are scored from one, meaning the patient is totally dependent on help to perform a task, to seven, meaning the patient is fully independent regarding the graded performance function. The motor scale grades the following tasks: 1) eating, 2) grooming, 3) bathing, 4) dressing-upper body, 5) dressing-lower body, 6) toilet, 7) bladder management, 8) bowel management, 9) transferring to bed, chair, wheelchair, 10) transferring to toilet, 11) transferring to tub and shower, 12) ability to walk or use a wheelchair, and 13) ability to climb stairs. The cognitive scale includes the following: 14) comprehension, 15) expression, 16) social interaction, 17) problem-solving, and 18) memory. The possible score range is $18-126$. In a previous study, we demonstrated that the FIM is a valid and reliable measure of 
patient functions in SCT setting.14 In our unit, FIM scores are assessed weekly by physiotherapists during the admission period.

\subsection{Data Collection Using A Multistate Model}

When applying GAMs, the multistate model is usually used for data collection to avoid overlearning. In the multistate model, some events are defined as states, and candidate predictor covariates on the day of each state are collected as representative data. In this study, we determined the following to be candidates of predictor covariates: Laboratory data, including the serum albumin (Alb) (mg/dL), alanine aminotransferase (ALT; U/L), and C-reactive protein (CRP; mg/dL) levels, blood sugar (BS; mg/dL), creatinine (Cre; mg/dL), and hemoglobin (Hb) levels (g/dL); white blood cell count (WBC; /microL), and physical data, including body temperature (BT; degrees Celsius), blood pressure (BP; mmHg), body weight (kg), DAS, and FIM scores. We determined the following events as states in our multistate model: 1) diarrhea, 2) need for oxygen supply, 3) hemorrhagic cystitis, 4) skin GVHD, 5) encephalitis, and 7) disease relapse. In addition to the days of occurrence or the seven abovementioned states, data regarding the predictor variables on the following day were collected at 2 weeks before SCT, the day of SCT, the day when SL occurred in the SL group, and the day of discharge in the control group. Data were allocated as blank if these states did not occur. An example data set from this study is shown below:

\begin{tabular}{|l|c|r|r|r|r|r|r|r|r|r|r|r|r|}
\hline Patient No. 1 & post-transplant day & Alb & Alt & CRP & BS & Cre & Hb & WBC & BT & BP & BW & DAS & FIM \\
\hline 2 weeks before & -14 & 3.7 & 14 & 0.8 & 107 & 0.68 & 8 & 29.6 & 36.7 & 110 & 76.8 & A & 108 \\
\hline SCT day & 0 & 3.2 & 34 & 0.6 & 160 & 0.59 & 8.8 & 0.5 & 37.1 & 110 & 75.7 & A & 108 \\
\hline diarrhea & 0 & 3.2 & 34 & 0.6 & 160 & 0.59 & 8.8 & 0.5 & 37.1 & 110 & 75.7 & A & 108 \\
\hline need for oxygen & 44 & 2.4 & 18 & 21.9 & 222 & 1.11 & 7.8 & 7.8 & 37.4 & 95 & 70.1 & B & 87 \\
\hline hemorrhagic cystitis & 7 & 3 & 43 & 0.6 & 153 & 0.57 & 7.6 & 0.2 & 36.8 & 115 & 74.4 & A & 108 \\
\hline skin GVHD & - & & & & & & & & & & & & \\
\hline encephalitis & - & & & & & & & & & & & & \\
\hline relapse & 39 & 2.4 & 19 & 7 & 166 & 1.32 & 8 & 25.8 & 37.9 & 90 & 68.5 & B & 107 \\
\hline skin trouble & 46 & 2.3 & 14 & 24.1 & 136 & 1.17 & 7 & 0.9 & 38.7 & 105 & 70.2 & B & 87 \\
\hline
\end{tabular}

An Example of data set (Patient No. 1) for multistate and GAM analysis

\subsection{Statistical Analyses}

Student's t-test was used for the comparison of FIM scores between the SL and control groups. P-values of $\leqq 0.05$ were considered significant. In the Picture-Frustration study (P-F study), the least significant difference (lsd) method was used for analysis; this is described below in the P-F study section. GAMs were applied to develop a regression equation predictive of the probability of SL development. In the GAMs, the P-value cut-off was defined as 0.1 to extract as many potential factors as possible.

\subsection{Theoretical Basis of GAM}

A Cox proportional-hazards model, based on the relationship between survival and the patient characteristics observed when the patient entered the study, was initially proposed. Because the values of the covariates changed over the course of the study, however, a number of theoretical problems with respect to the baseline survival function and baseline cumulative hazard function required solutions. Several prognostic models for the analysis of survival data with time-dependent covariates have become as widely used as the Cox proportional-hazards model.15,16 The present study examines the non-linear effects of covariate evolution over time, using penalized smoothing splines. The Cox proportional-hazards model postulates that the hazard at time $t$ is the product of two components:

$h(t)=h_{0}(t) \exp \left[\sum_{i=1}^{I} b_{i} x_{i}\right]$ 
American Research Journal of Nursing, Volume 1, Issue 3, August 2015

ISSN 2379-2922

where $b=\left(b_{1}, \cdots, b_{I}\right)$ is a vector of the coefficients. The proportional hazards assumption is that the baseline hazard $h_{0}(t)$ is a function of $\mathrm{t}$, but does not involve values of the covariates. Several prognostic models for heart transplant survival data have been developed using Cox regression analyses, and the values of all covariates were determined at the time when the patient entered the study.17 However, situations may exist in which the values of covariates change throughout the course of the study. A time-dependent model therefore, uses follow-up data to estimate the effect of covariates evolution over time. The relative hazard $h(t) / h_{0}(t)$ then depends on time t, and thus the proportional hazards assumption is no longer satisfied.16 By extending the GLM for the grouped data based on partial likelihood, a GLM can be proposed for ungrouped data,12,13 with time-dependent covariates for the discrete hazard rate $h_{l}^{<d>}$ of patient d at time-interval l:

$\mathrm{GLM}: \ln \left(\frac{h_{l}^{<d>}}{1-h_{l}^{<d>}}\right)=\beta_{0}+\beta_{1} \times($ days $)+\beta_{2} \times($ Alb $)+\beta_{3} \times($ Alt $)+\beta_{4} \times(C R P)+\beta_{5} \times(B S)+\beta_{6} \times($ Cre $)$

$+\beta_{7} \times(H b)+\beta_{8} \times(W B C)+\beta_{9} \times(B T)+\beta_{10} \times(B P)+\beta_{11} \times(B W)+\beta_{12} \times(D A S)+\beta_{13} \times(F I M)$

In recent years, a variety of powerful techniques have been developed for exploring the functional forms of effects. Here, a GAM with smoothing splines, which was proposed by Hastie et al.,18 will be used to extend the GLM as described by McCullagh et al.,19 the linear predictor in (1) is specified as the sum of the smooth functions s(x) with two continuously differentiable functions of some or all of the covariates:

$$
\begin{aligned}
& \mathrm{GAM}: \ln \left(\frac{h_{l}^{<d>}}{1-h_{l}^{<d>}}\right)=\beta_{0}+S(\text { days })+\beta_{1} \times(\text { Alb })+\beta_{2} \times(\text { Alt })+\beta_{3} \times(C R P)+\beta_{4} \times(B S)+\beta_{5} \times(\text { Cre }) \\
& +\beta_{7} \times(H b)+\beta_{8} \times(W B C)+\beta_{9} \times(B T)+\beta_{10} \times(B P)+\beta_{11} \times(B W)+\beta_{12} \times(D A S)+\beta_{13} \times(F I M) .
\end{aligned}
$$

A forward-selection method based on the deviance and corresponding number of degrees of freedom (d.f.) is given in the following supplementary table (The result of fitting GAM to the data). The deviance allows us to test the goodness-of-fit:

$$
\operatorname{Dev}=2\left(\ln L_{\max }-\ln L_{C}\right)
$$

where ${ }^{\ln L_{C}}$ denotes the maximized partial log-likelihood under some current GAM, and the log-likelihood for the maximum (full) model $\ln L_{C}$ is zero. The deviance exhibits an approximate $\chi 2$ distribution.

Supplementary table: The result of fitting GAM to the data

\begin{tabular}{|l|l|l|l|l|}
\hline Step & Terms fitted in model & Deviance & d.f. & p-value \\
\hline 0 & $S($ days $)$ & 159.471 & 371.274 & $<<0.00001$ \\
\hline 1 & $S($ days $)+D A S$ & 130.477 & 370.629 & $<<0.00001$ \\
\hline 2 & $S($ days $)+D A S+C R P$ & 124.086 & 369.660 & 0.01 \\
\hline 3 & $S($ days $)+D A S+C R P+A l b$ & 117.910 & 368.712 & 0.012 \\
\hline 4 & $S($ days $)+D A S+C R P+A l b+B S$ & 112.662 & 367.795 & 0.019 \\
\hline
\end{tabular}

We can thus obtain the final model: 
$\mathrm{GAM}: \ln \left(\frac{h_{l}^{<d>}}{1-h_{l}^{<d>}}\right)=\beta_{0}+S($ days $)+\beta_{1} \times(A l b)+\beta_{2} \times(C R P)+\beta_{3} \times(B S)+\beta_{4} \times(D A S)$.

\subsection{Picture-Frustration Study (P-F Study)}

In our unit, we routinely assess patients' psychosocial characteristics at admission using the P-F study and Yatabe-Guilford Personality (Y-G) study, as conducted by clinical psychologists. Because we recently reported that patients' characters were associated with the incidence of falls in a SCT setting,14 we compared the patients' characteristics in the ST and control groups using the P-F study and Y-G test. We investigated whether these data also correlated with a tendency toward SL development. The P-F study was originally designed to measure reactive aggressive behaviors in adults.20 All SCT patients in our SCT unit undergo the P-F study upon admission to the hospital. Clinical psychologists interpret patient responses according to nine scoring factor patterns: E' (extrapeditive), E (extrapunitive), e (extrapersistive), I' (intropeditive), I (intropunitive), i (intropersistive). M' (impeditive), $\mathrm{M}$ (impunitive), and $\mathrm{m}$ (impersistive); these patterns are based on three types of aggression (obstacle-dominance, ego-defense, and need-persistence), and three directions of aggression (others, self, and nobody). Using the least-significant difference method, we first applied a two-way analysis of variance (ANOVA) using two patient group factors (control and SL) and the nine patient character patterns (E', E, e, I', I, i, M', M, and m). If ANOVA detected a significant difference among the character patterns, Student's t-test was applied independently tor each character pattern to compare the difference in the average scores of the control and SL groups.

\subsection{Yatabe-Guilford Personality Test (Y-G Test)}

The Y-G test is a 120-question tool used to assess personality types and personality traits. The answer for each question is scored on a five point scale (lowest, lower, medium, higher, and highest) and evaluates personal traits such as "lack of cooperativeness." In this study, the $\chi^{2}$ test was used to compare points between the control and SL groups.

\subsection{Ethical Considerations}

This study was approved by the Institutional Review Board of the Hyogo College of Medicine and conducted according to the principles of the Declaration of Helsinki

\section{RESULTS}

\subsection{Patient Characteristics}

The patients included in this study are summarized in Table. 1. Among the 81 patients, 28 were in the SL group and 53 were controls. The distributions of age, sex, underlying disease, and donor type were nearly identical between the SL and control groups. We therefore shifted our focus to the time-dependent covariates affecting SL development.

A total of 81 patients were eligible for this study. Among these patients, 28 developed skin lesions (SL group), and the remaining 53 did not (control group). ALL: Acute lymphoblastic leukemia, including four patients with acute mixed lineage leukemia. Others: One case each of adult T-cell leukemia, chronic myelogenous leukemia, myeloma, and myeloproliferative disorder, and two cases of severe aplastic anemia. P-values for the distributions of age, sex, underlying disease, and donor type in the ST and control groups were calculated using the Student's t-test (age) or the $\chi^{2}$ test (sex, underlying disease, and donor type).

\subsection{Incidence of Each Event Defined as a State in the Multistate Model}


The multistate model is usually used in association with GAMs to avoid overlearning. Before establishing the predictive model using GAMs, we evaluated the incidence of the following events defined as states in the multistate model: 1) diarrhea, 2) need for oxygen supply, 3) hemorrhagic cystitis, 4) skin GVHD, 5) encephalitis, and 6) disease relapse. Among them, the occurrence rates of diarrhea, need for oxygen supply, and hemorrhagic cystitis were significantly higher in the SL group than in the control group. In particular, all SL group patients experienced diarrhea (Fig. 1).

Table1. Patients and characteristics.

\begin{tabular}{|c|c|c|c|c|c|}
\hline & & total & ST group & control group & \multirow[t]{2}{*}{ P-value } \\
\hline \multicolumn{2}{|l|}{ Number } & 81 & 28 & 53 & \\
\hline \multicolumn{2}{|c|}{ Age (median) } & $16-70(38)$ & $17-65(44)$ & $16-70(34)$ & \multirow{3}{*}{$\begin{array}{l}0.567 \\
0.138\end{array}$} \\
\hline $\operatorname{Sex}(M /$ & & $40 / 44$ & $17 / 11$ & $23 / 30$ & \\
\hline \multirow[t]{4}{*}{ Disease } & MDS/AML & $11 / 28$ & $4 / 8$ & $7 / 20$ & \\
\hline & ALL & 24 & 9 & 15 & \multirow{3}{*}{0.615} \\
\hline & ML & 12 & 6 & 6 & \\
\hline & others & 6 & 1 & 5 & \\
\hline \multirow[t]{4}{*}{ Donor } & related $(0-1 \mathrm{Ag})$ & 1 & 1 & 6 & \multirow{4}{*}{0.414} \\
\hline & related (haplo) & 25 & 25 & 39 & \\
\hline & unrelated & 1 & 1 & 5 & \\
\hline & CB & 1 & 1 & 3 & \\
\hline
\end{tabular}

Diarrhea, need for oxygen supply, hemorrhagic cystitis, skin GVHD, encephalitis, and disease relapse were defined as states in the multistate model. Data on the day when each event occurred were used in the GAM calculation; these are shown in Figs. 3, 4, and 5. The black and gray bars denote the SL and control groups, respectively.

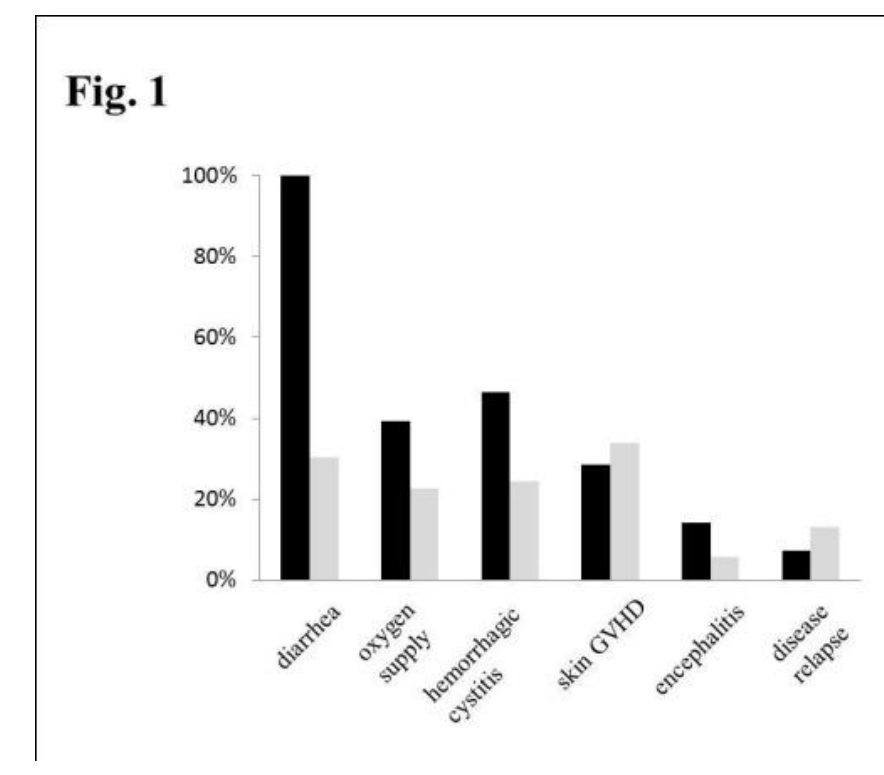

Fig1. Incidence of events defined as states in the multistate model in the skin lesion (SL) and control groups. 


\subsection{Correlation Between the DAS and FIM Score, and the Relationship of Each Score With SL Development}

In accordance with previous studies, the GAMs revealed patient activity affects SL development. The DAS significantly affected SL development in both the univariate and multivariate models. The FIM score however, was a significant factor in the univariate but not in the multivariate models, likely because the DAS, which correlated strongly with the FIM score (Fig. 2a,) and more comprehensively represents patient activity, was selected in the multivariate model. The FIM score is, however, a continuous variable that enables us to quantitatively assess a patient's physical ability. Accordingly, we compared the FIM scores of the SL and control groups, and found the SL group had significantly lower FIM scores relative to the control group every week after SCT (Fig. 2b).

The DAS and FIM scores correlated strongly $(\mathrm{P}<0.0001)$, which may have resulted in multicolinearity in the multivariate analysis. As a result, the FIM score was abandoned in the multivariate analyses.

The FIM scores of patients in the SL group were significantly lower than those of patients in the control group every week (0-5 weeks) after SCT.

\section{Fig. 2a}

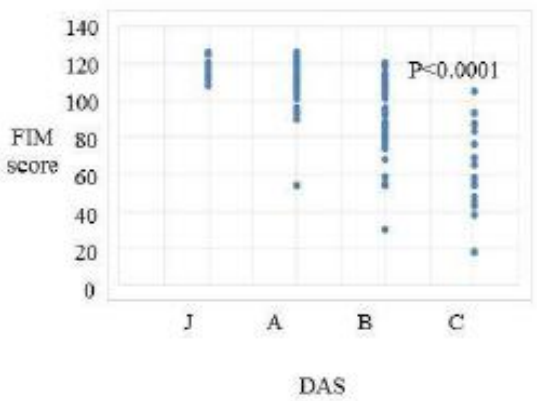

Fig. 2b

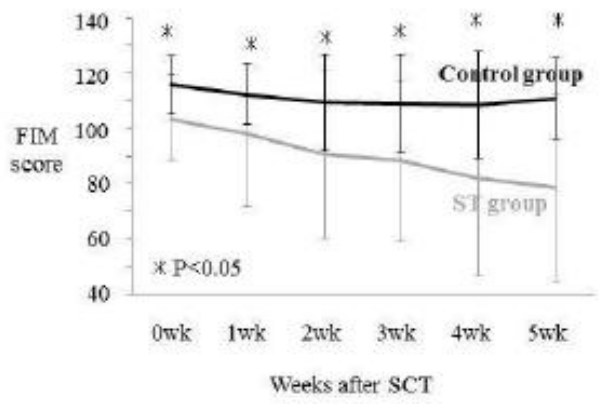

Fig2a. Correlation between the daily activity score (DAS) and functional independence measure (FIM) score.

Fig2b. Comparison of functional independence measure (FIM) scores in the skin lesion (SL) and control groups after stem cell transplantation (SCT.)

\subsection{Prediction of SL Development Using Gams}

A logistic regression model based on data from the multistate model was established using GAMs. Candidate predictive covariates comprised laboratory data, including the serum levels of Alb, ALT, CRP, BS, Cre, Hb, and WBC, as well as physical data, including BT, BP, body weight, DAS, and FIM scores. Among these, Alb, BS, DAS, and the post-transplant day were significant factors $(\mathrm{p}<0.1)$ predictive of SL development (Fig. 3). Whereas Alb, BS, and DAS exhibited linear curves, the post-transplant day exhibited a reversed U-shaped curve, indicating the most susceptible time for SL development was approximately day 30 (Fig. 4). Figure 5 shows a comparison of the predictive efficacy of SL development, thus demonstrating that GAMs, but not GLMs, represent the reality of SL development.

After selecting the optimum smoothing parameters, the deviance (3) of our data was $\operatorname{Dev}=131.072\left(<\chi_{367.795}^{2}\right)$. In other words, our model was fairly well fitted. 
A regression equation to determine the odds of SL development, represented by logit (p), was deduced using GAMs. The right side of the equation comprises the serum albumin level (Alb) (mg/dL), serum glucose level (BS) (mg/dL), daily activity score (DAS), and post-transplant day. This equation suggests SL development is associated with the nutritional status (Alb), BS control (BS), patient activity (DAS), and the susceptible time (post-transplant day). S (day) denotes a spline function of the post-transplant day, which is illustrated in Figure 4.

Fig. 3

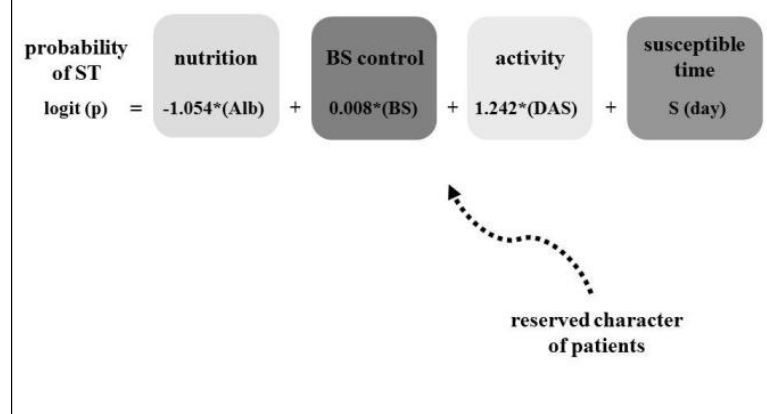

Fig3. Schema showing the prediction of skin lesion (SL) development.

Fig. 4

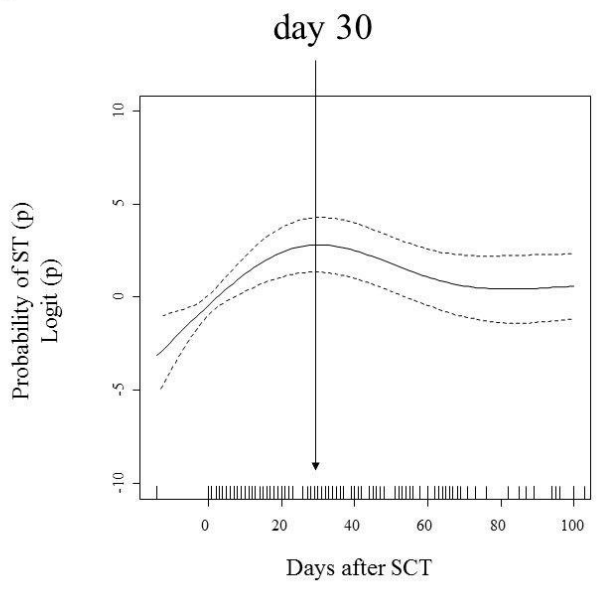

Fig4. Spline curve of the post-transplant day.

The post-transplant day exhibited a non-linear pattern. The spline curve of the post-transplant day shows a reversed U-shaped pattern with a peak at approximately post-transplant day 30.

An example prediction of SL development using GLMs (open triangle) and GAMs (closed circle), respectively, in a patient who actually developed SL after achieving several states. The vertical axis denotes the probability of not developing SL. Whereas GLM could not predict SL development, GAM predicted the occurrence of SL on day 19. 


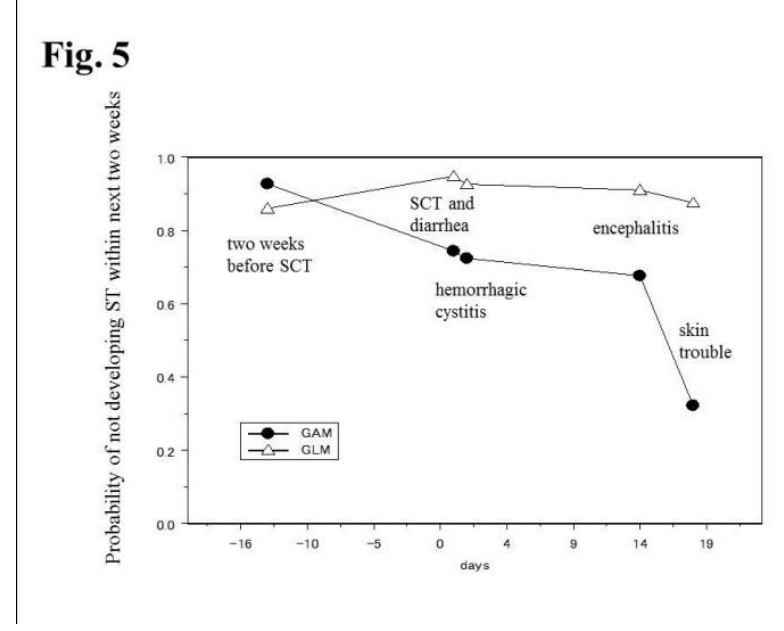

Fig5. Comparison of generalized linear models (GLMs) and generalized additive models (GAMs) for predicting skin lesion (SL) development.

\subsection{Patient Characteristics and SL Development}

Finally, we sought another factor that would affect SL development. Notably, psychosocial aspects may affect patient behavior. We compared patient characteristics in the SL and control groups using the P-F study and Y-G test. In the P-F study, significant differences were observed with respect to patients' character patterns. With regard to a comparison of the character differences between the groups, the control group had a higher score only for the E' pattern, which is thought to represent a person who tends to directly express their complaints or pain. In the Y-G test, there were no differences in personality types between the control and SL groups, although for the trait of "lack of cooperativeness," the control group had higher points than the SL group. Taken together, patients in the SL group were self-repressive, and had a tendency to resolve problems on their own, whereas patients in the control group had a tendency to express their discomfort. This reserved character in SL group patients likely delayed the discovery of signs leading to SL development, resulting in a high incidence of SL.

\section{DISCUSSION}

Patients who have undergone SCT are prone to many complications. Diarrhea because of gut GVHD and/or other pathogeneses is among the most common manifestations after SCT. Following SCT, patients suffer from perianal skin erosion because of fecal incontinence, in addition to pure pressure ulcers, which predominantly occur at bony prominences and are caused by pressure, shear, or friction. The classification of wounds in the gluteal cleft and buttocks has been challenging, even for wound care experts.21 To avoid confusion regarding this classification and to meet the needs of clinical situations in SCT settings, we have used the more comprehensive term SL and have investigated the risk factors affecting the occurrence of SL.

A number of published reports have focused on pure pressure ulcers, and several scales predicting the development of these ulcers have been established, such as the Braden scale,22 Norton scale,23 Modified Norton scale,24 Waterlow scale, 9 and Glamoran scale.5 The Braden scale comprises six items: Activity, mobility, nutritional status, sensory perception, friction and shear, and moisture. Whereas the Norton scale comprises five items: General physical condition, mental status, activity, mobility, and moisture, the Modified Norton scale includes the items of nutritional status and fluid intake, in addition to the Norton scale items. Although the adopted items of these scales 
differ somewhat, daily activity is a crucial factor associated with SL development. In our study, both the DAS and FIM score, which were mutually correlated, were extracted as significant covariates for SL development. The DAS remained a significant covariate in the multivariate analysis, likely because it more comprehensively represents a patient's condition. The FIM score, on the other hand, is a continuous variable, and should be useful in quantitative comparisons between patients in the SL and control groups. In this study, patients in the SL group had lower FIM scores than those in the control group both before and after SCT.

Regarding other comorbid conditions, higher BS levels, higher serum pH levels, and a lower diastolic BP were reported to be predictors of pressure ulcers in subjects receiving mechanical ventilation.7 In our study, a high BS levels was a significant covariate for SL development, whereas an average BP was not. We found the serum Alb level was a significant predictor for SL development, suggesting the nutritional status is also a crucial factor in SL development, in accordance with previous scales.24 Finally, the post-transplant day was found to be another factor for SL development, and was shown to exhibit a non-linear curve. In contrast to GLMs, which have hitherto been used to analyze various types of clinical data, GAMs can respond to non-linear variables, and have recently been applied in the life science field. A strong merit of this work is that by applying GAMs, we were able to establish a regression model predictive of SL development, using the serum Alb, BS, DAS, and post-transplant day.

Regarding the psychosocial aspect, whereas patients in the control group tended to express their discomfort, patients in the SL group were self-repressive, and tended to resolve problems by themselves. This reserved character observed in the SL group patients likely delayed the discovery of signs leading to SL development, resulting in a high incidence of SL.

To date, GAMs have been used in only one report in the SCT field.25 Using our models, nurses can predict the probability of SL development within 2 weeks in real time. However, this study was limited by the low number of samples from which to detect new risk factors for SL development. A validation study involving a large number of patients would be needed to determine new risk factors and confirm the results of this study.

In conclusion, the development of SL in patients after SCT was associated with and predicted by covariates such as serum Alb, BS, DAS, and post-transplant day. Although the post-transplant day exhibited a non-linear curve, GAMs could accurately integrate these parameters into a regression model. Furthermore, a reserved psychosocial character may be associated with susceptibility to SL.

Authors' contributions: S.U. and K.I. designed the study and wrote the manuscript, S.U. and Y.T. collected the data, M.T. performed the statistical analyses, R.M. was responsible for psychological issues, K.K. and T. I. contributed to clinical management, and J. M. and H.O. supervised the project.

The authors declare no conflicts of interest.

\section{REFERENCES}

[1] Jones D. Pressure ulcer prevention in the community setting. Nurs Stand 2013; 28: 47-55.

[2] Dellefield ME, Magnabosco JL. Pressure ulcer prevention in nursing homes: Nurse descriptions of individual and organization level factors. Geriatr Nurs 2014; 35: 97-104.

[3] Sving E, Idvall E, Högberg H, Gunningberg L. Factors contributing to evidence-based pressure ulcer prevention. A cross-sectional study. Int J Nurs Stud 2014; 51: 717-25.

[4] McGuinness J, Persaud-Roberts S, Marra S, et al. How to reduce hospital-acquired pressure ulcers on a neuroscience unit with a skin and wound assessment team. Surg Neurol Int 2012; 3: 138.

[5] Kottner J, Kenzler M, Wilborn D. Interrater agreement, reliability and validity of the Glamorgan Paediatric Pressure Ulcer Risk Assessment Scale. J Clin Nurs 2014; 23: 1165-9. 
[6] Balzer K, Kremer L, Junghans A, Halfens RJ, Dassen T, Kottner J. What patient characteristics guide nurses' clinical judgement on pressure ulcer risk? A mixed methods study. Int J Nurs Stud 2014; 51: 703-16.

[7] Senturan L, Karabacak U, Ozdilek S, et al. The relationship among pressure ulcers, oxygenation, and perfusion in mechanically ventilated patients in an intensive care unit. J Wound Ostomy Continence Nurs 2009; 36: 503-8.

[8] Da Silva D, Han L, Faivre R, Costes E. Influence of the variation of geometrical and topological traits on light interception efficiency of apple trees: sensitivity analysis and metamodelling for ideotype definition. Ann Bot 2014 Apr 10. [Epub ahead of print]

[9] Thelen B, French NH, Koziol BW, et al. Modeling acute respiratory illness during the 2007 San Diego wildland fires using a coupled emissions-transport system and generalized additive modeling. Environ Health 2013; 12: 94.

[10] You YA, Ali M, Kanungo S, et al. Risk map of cholera infection for vaccine deployment: the eastern Kolkata case. PLoS One 2013; 8: e71173.

[11] Hung CY, Sun PL, Chiang SJ, Jaw FS. In vitro differential diagnosis of clavus and verruca by a predictive model generated from electrical impedance. PLoS One 2014; 9: e93647.

[12] Tsujitani M, Sakon M. Analysis of survival data having time-dependent covariates, IEEE Transaction on Neural Networks 2009; 20: 389-394.

[13] Tsujitani M, Tanaka Y, Sakon M. Survival analysis with time-dependent covariates using generalized additive models, Computational and Mathematical Methods in Medicine, 2012, Article ID 986176.

[14] Ueki S, Kozawa M, Miyamoto J, Mori R, Ogawa H, Ikegame K. Risk analysis of falls in patients undergoing allogeneic hematopoietic stem cell transplantation. Clin J Oncol Nurs 2014; 18: 396-9.

[15] Murtaugh PA, Dickson ER, Van Dam GM, et al. Primary biliary cirrhosis: prediction of short-term survival based on repeated patient visits. Hepatology 1994; 20: 126-34.

[16] Altman DG, De Stavola BL. Practical problems in fitting a proportional hazards model to data with updated measurements of the covariates. Stat Med 1994; 13: 301-41.

[17] Aitkin M, Laird N, Francis B. A reanalysis of the Stanford Heart Transplant data. J. Am. Statist. Assoc 1983; 78: $264-292$.

[18] Hastie TJ, Tibshirani, RJ. Generalized Additive Models, Chapman and Hall: London, 1990.

[19] McCullagh P, Nelder JA. Generalized Linear Models, 2nd edn. London; Chapman and Hall, 1989.

[20] Rosenzweig S. The picture-association method and its application in a study of reactions to frustration. J Pers 1945; 14: $3-23$.

[21] Mahoney M, Rozenboom B, Doughty D. Challenges in classification of gluteal cleft and buttocks wounds: consensus session reports. J Wound Ostomy Continence Nurs 2013; 40: 239-45.

[22] Braden B, Bergstrom N. A conceptual schema for the study of the etiology of pressure sores. Rehabil Nurs 1987; 12 : 8-12.

[23] Norton D, McLaren R, Exton-Smith AN. An investigation of geriatric problems in hospital, $3^{\text {rd }}$ edn. Churchill Livingstone: London, 1979.

[24] Ek AC, Unosson M, Larsson J, Von Schenck H, Bjurulf P. The development and healing of pressure sores related to the nutritional state. Clin Nutr 1991; 10: 245-50.

[25] Couper D, Pepe MS. Modelling prevalence of a condition: chronic graft-versus-host disease after bone marrow transplantation. Stat Med 1997; 16: 1551-71. 